\title{
Ratio of bcl-xshort to bcl-xlong Is Different in Good- and Poor-Prognosis Subsets of Acute Myeloid Leukemia
}

\author{
G. Deng, ${ }^{1}$ C. Lane, ${ }^{1}$ S. Kornblau, ${ }^{1}$ A. Goodacre, ${ }^{1}$ V. Snell, ${ }^{1}$ \\ M. Andreeff, ${ }^{1}$ and A. B. Deisseroth ${ }^{2}$ \\ ${ }^{1}$ University of Texas M. D. Anderson Cancer Center, Houston, Texas, U.S.A. \\ ${ }^{2}$ Yale University School of Medicine, New Haven, Connecticut, U.S.A.
}

Communicated by E. Beutler. Accepted January 9, 1998.

\begin{abstract}
Background: Acute myeloid leukemia (AML) is a heterogeneous collection of leukemic disorders ranging from chemotherapy-sensitive subsets [inversion 16 and $t(8 ; 21)]$, which often can be cured with cytosine arabinoside alone, to the most resistant subsets, which can survive even supralethal levels of combination alkylator chemotherapy (cytogenetic subsets monosomy 5 and monosomy 7).

Materials and Methods: To analyze the expression of $B C L-2$ family genes, which are expressed in these subsets of AML, we used PCR sequence amplification reactions that are dependent on oligonucleotide primers representing the $\mathrm{BH} 1$ and $\mathrm{BH} 2$ homology domains to generate the unique regions between $\mathrm{BHI}$ and $\mathrm{BH}$. These primers are conserved among all members of the $B C L-2$ gene family and are separated by a 150 nucleotide region sequence between the $\mathrm{BH} 1$ and $\mathrm{BH} 2$ domains. The PCR products unique to each $B C L-2$ family member were cloned directionally into sequencing vectors. The identity of the insert of each clone was determined by slotblots of the DNA amplified from individual colonies and by hybridization with radioactive probes specific to the bcl-2, bcl-x, or bax genes.
\end{abstract}

Results: We found that bcl- 2 is the predominant member expressed in AML samples with a poor prognosis $(-5,-7)$, whereas the transcripts of bcl-x are higher than those of bcl-2 in the AML samples with a good prognosis [inv16, $t(8 ; 21)]$. No significant difference in bax expression was detected between AML subsets of good and bad prognosis. The ratio of bcl-xlong, which inhibits apoptosis, to bcl-xshort, which promotes apoptosis, was determined by amplification with a pair of primers specific to bcl-x followed by separation of the PCR product on agarose gels. Bcl-xlong and bcl-xshort appeared as bands of different molecular mass on a molecular weight gel and were visualized by ethidium bromide staining or Southern blot analysis with a bcl-x-specific probe.

Conclusions: We found that the ratio of bcl-x long to bcl-x short was higher in the AML patients with a poor prognosis. These experiments showed that the levels of $B C L-2$ family members in the leukemia cells of good- and poor-prognosis subsets are different. In addition, novel members of the $B C L-2$ family were isolated from the cells of AML patients of either prognosis.

\section{Introduction}

Cytogenetic analysis of acute myeloid leukemia (AML) cells has been used to predict the respon-

Address correspondence and reprint requests to: Dr. Albert Deisseroth, Yale University School of Medicine, Section of Medical Oncology, WWW221, 333 Cedar Street, New Haven, CT 06520-8032, U.S.A. Phone: 203-737-5608; Fax: 203-737-5698; E-mail: DEISSEROAB@MASPO2.MAS.YALE. EDU siveness of patients to induction chemotherapeutic regimens $(1,2)$. AML patients whose leukemia cells contain inversion 16 or $\mathrm{t}(8 ; 21)$ are more often than not sensitive to single-agent cytosine arabinoside treatment (3-8), whereas patients whose leukemia cells contain monosomy 5 , monosomy 7 , or trisomy 8 are resistant to even ablative levels of combination therapy $(6-8)$. The molecular changes associated with 
these chromosomal abnormalities are under intensive investigation (9-12). At the present time, the mechanisms of resistance or sensitivity are not completely understood.

Many neoplastic cells die by apoptosis when treated with DNA-damaging chemotherapeutic agents (13). The $B C L-2$ family of genes has been shown to contain members that either induce or prevent the apoptosis process (14-16). All of the $B C L-2$ gene family members share a high degree of amino acid sequence similarity in the $\mathrm{BHl}$ and $\mathrm{BH} 2$ regions, which are separated by a unique sequence region of approximately 50 amino acid residues. It has been shown that the bcl-2, bcl-xl, and mcl proteins inhibit cell death, whereas the bax, and bcl-xs proteins promote cell death (14,17-20). The $B C L-2$ family also includes recently identified genes such as $B A D, B A K, B A G$, the function of which remains to be tested (21-26).

Since the balance among the $B C L-2$ family members that inhibit or induce cell death appears to determine the survival of cells in certain circumstances (26), we investigated the expression profile of individual $B C L-2$ members in AML cells from the chemotherapy-sensitive and -resistant cytogenetic subtypes of AML. We tested the ratio between pro-apoptosis transcripts and anti-apoptosis transcripts for a possible association between the expression of specific $B C L-2$ genes and the response to chemotherapy. We also attempted to isolate novel $B C L-2$ family members from AML cells.

\section{Materials and Methods}

Cells

Cells were obtained from a repository of frozen pheresis samples from patients with AML. The samples had been obtained as incidental specimens that were collected according to protocols approved by the University of Texas M. D. Anderson Cancer Center Institutional Review Board. The results of cytogenetic analysis of these leukemia cells were retrieved from the medical record of each patient diagnosed with AML.

\section{Amplification of $c D N A$ by $R T-P C R$}

RNA was extracted with the TriZol Reagent from GibcoBRL. Poly-A plus RNA was extracted with the FastTrack Kit from InvitroGen. Oligonucleotides were synthesized by a Cruachem, Inc. oli- gonucleotide synthesizer. The sequences of the oligonucleotides are $5^{\prime}$-aactggggnmgsrtbgtsrc- $3^{\prime}$ and 5'-gcarcckccntkntbnhgbatcca-3'. Reverse transcription (RT) was carried out with the Superscript Preamplification System from GibcoBRL. Polymerase chain reaction (PCR) was carried out with the annealing temperature set at $50^{\circ} \mathrm{C}$.

\section{Slot-Blots and Southern Blots}

The Hybond-N+ nylon membrane from Amersham was used for slot-blot and Southern blot analysis. Radioactive probe was generated with the RediPrime Kit from Amersham Life Science. The hybridization was carried out in the Rapidhyb buffer from Amersham in a rotating hybridization oven. The stringency of the final washing was $0.2 \times \mathrm{SSC}, 0.1 \% \mathrm{SDS}, 60^{\circ} \mathrm{C}$ for $30 \mathrm{~min}$.

\section{Cloning of PCR Products}

The PCR products were cloned with the pCRTRAP plasmid system from GeneHunter. The transformed bacteria were allowed to grow on LB plates with $20 \mu \mathrm{g} / \mathrm{ml}$ tetracycline.

\section{Sequencing of PCR Products}

The sequences of the PCR products were determined by automatic DNA sequencing, using the plasmid DNA as templates, in the University of Texas M. D. Anderson Cancer DNA Sequencing Facility.

\section{Results} Amplification of $C D N A$ S Containing BHI/BH2
Domains from $A M L$ Cells

The $B C L-2$ gene family members share remarkable homology in the $\mathrm{BH} 1$ and $\mathrm{BH} 2$ domains $(14,15,26)$. These two homologous domains are interrupted by a 50 amino acid, 150 nucleotide sequence stretch that is unique for each member of the $B C L-2$ family. We designed a pair of oligonucleotides primers that represent the consensus nucleotide sequence of the $\mathrm{BH} 1$ and $\mathrm{BH} 2$ regions. Most importantly, the unique sequence region between $\mathrm{BH} 1$ and $\mathrm{BH} 2$ that can be synthesized using these primers, can be used to identify each gene of the family because the sequence in this region is different for each member of the $B C L-2$ gene family.

Poly-A plus RNA was extracted from each cytogenetic prognostic subset (both poor- and 


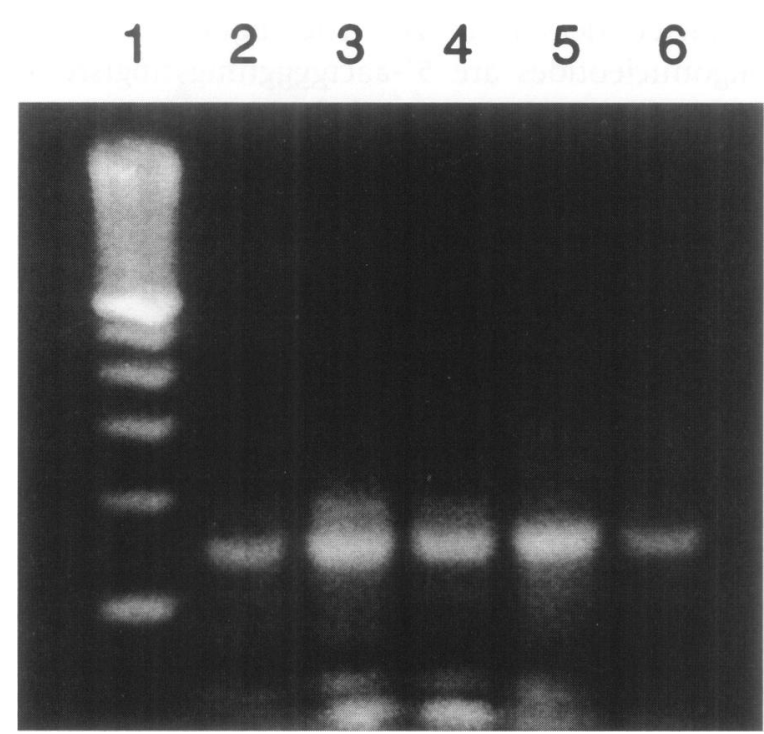

Fig. 1. PCR amplification of CDNAs containing BH1 and BH2 domains. Poly-A plus RNAs from AML cells with the indicated cytogenetic abnormalities were used as starting materials in RT-PCR reactions. The sequences of the primers were derived by synthesizing a family of oligonucleotides that contain the nucleic acid sequences of the $\mathrm{BH} 1 / \mathrm{BH} 2$ domains. The predicted size of PCR products from correct priming is approximately $150 \mathrm{bp}$. Lane 1 : MW marker (100 bp ladder). The cytogenetics of the cells in lanes 2-5 are monosomy 5, monosomy 7 , inversion 16 , and $t(8 ; 21)$, respectively. Plasmid DNA with bcl-2 insert served as the template in lane 6 as positive control.

good-prognosis AML) of the leukemia cells of patients with AML. The RNA served as template in a RT-PCR reaction, with the $5^{\prime}$-bcl and $3^{\prime}$-bcl sequences as primers. The PCR products were analyzed by $3 \%$ Nusieve agarose gel (Fig. 1). The predicted size of the $\mathrm{BH} 1 / \mathrm{BH} 2$ region was approximately $150 \mathrm{bp}$.

\section{Identification of Amplification Products}

The 150 bp $\mathrm{BH} 1 / \mathrm{BH} 2$ amplification products consisted of a mixture of $B C L-2$ family members and nonspecific sequences. To determine which individual family members are included among these amplification products and at what level of abundance these sequences are represented, we cloned the PCR products in the PCR-TRAP plasmid vector. Competent bacteria were transformed and plated on LB tetracycline plates, which offered a selection against self-ligated vectors that did not have inserts. In effect, we constructed cDNA mini-libraries representing the cellular transcripts that contain $\mathrm{BH} / \mathrm{BH} 2$-like sequences.

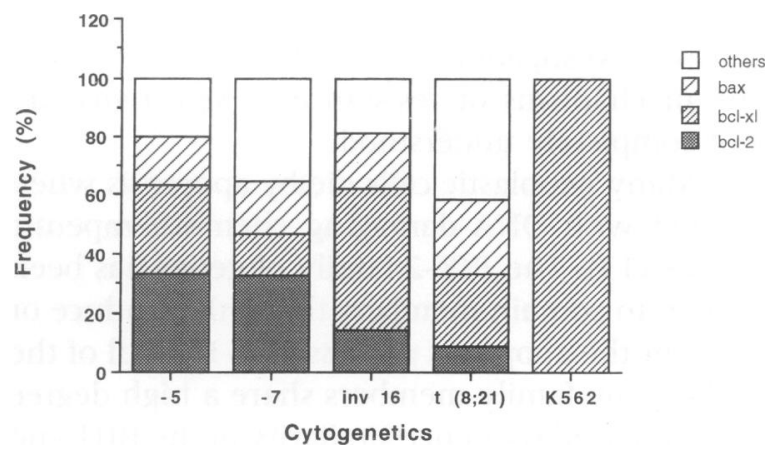

Fig. 2. Expression of bcl-2 family members in AML cells. The composition of the $\mathrm{BH} 1 / \mathrm{BH} 2$ amplification products was determined by cloning the PCR products followed by identification of the insert in each clone by slot-blot analysis of the bacterial colonies with probes specific to bcl-2, bcl-xl, and bax. The frequency of individual members was calculated by dividing the number of clones hybridized with a specific probe by the number of total clones containing the $150 \mathrm{bp}$ insert. The abbreviations for cytogenetics are: -5 , monosomy $5 ;-7$, monosomy 7 ; inv 16 , inversion $16 ; t(8 ; 21)$, translocation $8 ; 21$. K562 cells were used as a control.

Nearly 250 individual bacterial colonies from these mini-libraries were picked and used to inoculate LB broth in 96-well tissue culture plates. The resulting bacterial cultures were subsequently blotted on nylon membrane in a slotblot apparatus. The identities of the DNA inserts in each bacterial colony were determined by carrying out hybridization with radioactive probes specific to known members of the $B C L-2$ gene family, such as bcl-2, bcl-x, bax, and mcl. The number of clones hybridized with respective probes were counted.

Upon further analysis, some of the clones that did not hybridize to any of the probes were found to contain no inserts of $150 \mathrm{bp}$. They were removed from the analysis. The composition of each mini-library was constructed by counting the number of colonies that hybridized with the major $B C L-2$ family members. The results of these analyses are shown in Figure 2.

$\mathrm{Bcl}-2$ appeared to be the most abundantly expressed member of the $B C L-2$ gene family in the poor-prognosis AML subsets associated with monosomy 5 and monosomy 7 karyotypes. Interestingly, nearly $50 \%$ of the DNA fragments amplified from AML cells of the good-prognosis, inversion 16 subset hybridized to a bcl-xlong probe. The bax subsets were represented equally among all subsets. No mcl homologous products were detected in any of the AML samples. In the 


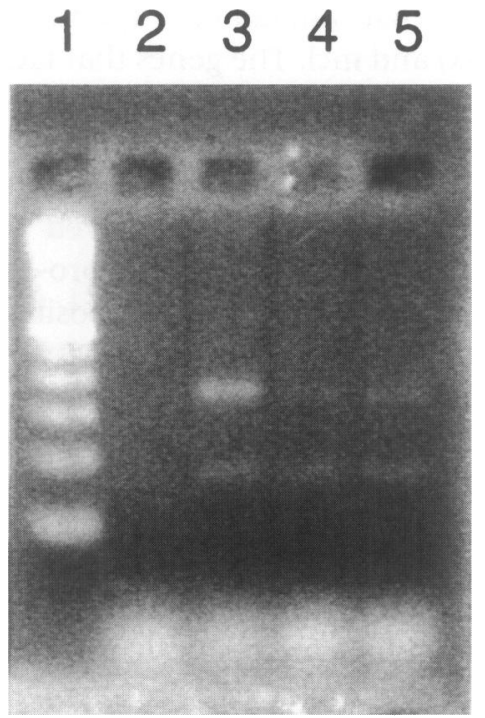

Fig. 3. Expression of bcl-xlong and bcl-xshort in AML cells. Bcl-x-specific primers were used to amplify the two species of bcl-x transcript from AML cells. The PCR products were visualized by agarose gel electrophoresis. The bcl-xlong and bcl-xshort products appeared as bands of different molecular weight as marked. Lane 1: MW marker (100 bp ladder). The cytogenetics of the cells in lanes $2-5$ are monosomy 5 , monosomy 7 , inversion 16 , and $t(8 ; 21)$, respectively.

control K562 cells, which contain the $B C R-A B L$ fusion gene, all 50 clones tested were homologous to the bcl-xlong probe.

\section{Examination of the Ratio between bcl-xlong and bcl-xshort Transcripts}

These two splicing variants of the bcl-x gene product are called bcl-xlong and bcl-xshort.
Since bcl-xlong is associated with resistance to apoptosis and bcl-xshort promotes apoptosis, it would be interesting to know the ratio between the two forms of bcl-x transcripts in the chemotherapy-sensitive and -resistant forms of AML, as they function in opposite ways for the regulation of cell death. The 5'-bcl and 3'-bcl primers used for the PCR reaction can only amplify the bclxlong cDNA; the $\mathrm{BH} 1$ and $\mathrm{BH} 2$ regions are deleted in bcl-xshort mRNA. Therefore, we designed a separate pair of primers that are specific to bcl-x. The RT-PCR products of bcl-xlong and bcl-xshort appeared as DNA fragments of different sizes on agarose gels when these primers were used.

As shown in Figure 3, bcl-xlong was expressed at a higher level than was bcl-xshort in samples from AML patients of the monosomy 5 and monosomy 7 subsets. In contrast, the ratio between bcl-xlong and bcl-xshort is lower in inversion 16 and $t(8 ; 21)$ subsets of AML cells.

To extend this observation to a larger number of patients, we extracted total RNA from additional samples from AML patients and carried out RT-PCR analyses with the bcl-x-specific primers to test whether the bcl-xlong or bclxshort subsets were dominant in the good- or poor-prognostic subsets of AML. The amount of RT-PCR products was too small to be visible by ethidium bromide staining. The PCR products were evaluated by Southern blot hybridization with radioactive bcl-x probes. The results of these analyses are shown in Table 1 . The ratio between bcl-xlong and bcl-xshort splicing variants is higher in samples from the AML patients with poor-prognosis cytogenetics than in the

Table 1. Southern blot analysis of PCR-amplified bcl-xlong and bcl-xshort products from AML cells

\begin{tabular}{cclccl}
\hline Patient & bclxl/bclxs & Cytogenetics & Patient & bclxl/bclxs & Cytogenetics \\
\hline SN & 5.0 & -7 & AE & 1.9 & t8;21 \\
SS & 2.7 & +8 & FY & 1.8 & $+22,+8$,inv16 \\
HS & 2.2 & -7, inv3 & BE & 1.6 & +8 \\
SR & 2.2 & $-9 q, \mathrm{t} 8 ; 21$ & MY & 1.6 & t8;21 \\
HT & 2.1 & $-5-7$ & EE & 1.3 & inv16 \\
LE & 1.9 & $-5-7$ & HE & 1.3 & inv16 \\
LN & 1.9 & $-5-7$ & TE & 1.2 & $\mathrm{t} 8 ; 21$
\end{tabular}

RT-PCR was carried out with total RNA as templates and bcl-x specific oligonucleotides as primers. The PCR products were separated by agarose gel electrophoresis, transfered to nylon membrane in alkaline solution and hybridized with radioactive probes derived from bcl- $x$ cDNA. The filter was exposed to multiple X-ray films. The ratio between the bcl-xlong signal and bcl-xshort signals was calculated from the image intensity on the films. 
samples from AML patients with good-prognosis cytogenetic markers.

\section{Analysis Testing for Presence of Novel Genes that Belong to the bcl-2 Gene Family}

Thirty-two clones were identified from the AML PCR reactions, the products of which did not hybridize to the probes for known members of the $B C L-2$ family (bcl-2, bcl-x, bax, and mcl). These clones, however, were shown to contain 150 nucleotide unique sequence regions between the two $\mathrm{BH} 1$ and $\mathrm{BH} 2$ homology regions. It is possible that these clones may contain sequences for previously unidentified members of the $B C L-2$ family. Plasmid DNA was therefore prepared from these products and used as a template in dideoxynucleotide sequencing reaction. The sequences were submitted to the GenBank databases for homology search by the BLAST programs.

Two clones of particular interest were identified which we designated bcln l and bcln9. Bclnl contained sequences homologous to the polycystic kidney disease protein. No homology for the nucleic acid and peptide sequences of bcln9 was found after extensive search in various databases with all available algorithms. Fulllength cDNAs of bclnl and bcln9 are being obtained and are subject to further analysis.

\section{Discussion}

Leukemia cells collected from patients with AML show a variety of cytogenetic abnormalities. Some of these chromosomal aberrations are associated (inversion 16 and $t(8 ; 21)$ ) with excellent response to chemotherapy. AML patients with karyotypes of inversion 16 or $t(8 ; 21)$ are very sensitive to cytosine arabinoside as a single agent. These cells may have an unusual sensitivity to cytosine arabinoside which may exceed that of the normal myeloid cells. On the other hand, cells from the AML patients of the monosomy 5 or monosomy 7 subtypes are notoriously resistant at diagnosis even to intensive systemic combination chemotherapy. Making the resistant cells respond to chemotherapeutic agents remains a challenge in the treatment of AML.

A number of studies suggested a role of the $B C L-2$ gene family in the regulation of chemoresistance in AML (13). The $B C L-2$ family is an expanding group of genes that shares the highly conserved $\mathrm{BH} 1$ and $\mathrm{BH} 2$ domains. Members of the family that antagonize apoptosis include bcl-2, bcl-xl and mcl. The genes that facilitate cell death include bax, bcl-xshort, and bak. It has been shown that these gene products can form homo- or heterodimers with each other. The regulation of cell death is achieved in part by varying the ratio between the pro- and antiapoptosis members in the composition of the dimers, which can alter the sensitivity of the cells to DNA damaging agents.

We therefore tested the expression of the $B C L-2$ gene family members in AML cells with representative karyotypes of these good- and poor-prognosis subsets of AML. We were especially interested in the ratio among the expression of individual members. It was shown that the ratio between pro- and anti-apoptosis molecules is more important than the absolute amount of gene products in determining cellular susceptibility to apoptosis. We also looked for an association between the expression pattern and sensitivity to chemotherapy.

By using oligonucleotides representing the $\mathrm{BH} 1$ and $\mathrm{BH} 2$ domains, we were able to amplify all transcripts that contained $\mathrm{BH} 1-\mathrm{BH} 2$ intervening regions. Among them were known $B C L-2$ family member clones that may be associated with previously unidentified members of the $B C L-2$ gene family. We determined the ratio among various expression products by analyzing the composition of the PCR products and separating these products on a molecular weight gel. As shown in Figure 2, bcl-2 appeared to be the most abundant member expressed in samples from the poor-prognosis AML group, whereas it was a minor component in samples from the good-prognosis group.

It has been reported that there was a significantly greater level of bcl-2 expression in cells from AML patients who failed to achieve remission after chemotherapy than in those who responded (27). It was also shown that inhibition of bcl-2 with antisense oligonucleotides increased the sensitivity of AML blasts to cytosine arabinoside (28). Our studies suggested that the higher level of bcl-2 expression may contribute to the poor response to chemotherapy observed in AML in patients whose leukemia cells have the monosomy 5 or monosomy 7 cytogenetic abnormality.

The molecular approach used in Figure 2 could not discriminate among the alternative splicing transcripts bcl-xshort from the bcl-xlong variant of the bcl-x gene. Higher levels of bclxlong expression could protect against apoptosis 
by bcl-xshort. We therefore examined the ratio between the two forms of bcl-x transcript by PCR amplification of bcl-x cDNA with a pair of bcl-xspecific primers. As shown in Figure 3 and Table 1, the ratio between the anti-apoptotic bclxlong and the pro-apoptotic bcl-xshort is higher in cells from the chemoresistant subtypes than in cells from the chemosensitive subtypes. Bclxlong has been demonstrated to be able to confer resistance to a variety of cytotoxic drugs including cisplatin, etoposide, vincristine, and hygromycin B $(29,30)$. We also noted that bcl-xlong appeared to be the single most abundant $B C L-2$ family member in the $\mathrm{K} 562$ cell line which is known to be resistant to many chemotherapeutic agents (Fig. 2).

Our strategy of amplifying all cDNA-containing regions homologous to $\mathrm{BH} 1$ and $\mathrm{BH} 2$ domains offered an opportunity to test for the presence of or to isolate clones for new $B C L-2$ family members from AML cells. The clones shown in Figure 2 as "others" may include such members. Sequence analysis revealed that some of the clones seemed to come from less specific priming during PCR. The remaining clones represent $\mathrm{CD}$ NAs with previously unidentified functions. Their membership in the $B C L-2$ family and their involvement in the modulation of apoptosis will be determined in further analysis.

In summary, we studied the expression profiles of $B C L-2$ family members in AML cells from different cytogenetic and prognostic subgroups. Previous studies usually focused on the expression of individual members without looking at all members in one study. The results of our analysis suggest that an association exists between the ratio of anti-apoptotic to pro-apoptotic members of the $B C L-2$ gene family and the chemoresistance of AML cells. These observations may ultimately lead to molecular interventions in AML that are designed to sensitize the cells to chemotherapy.

\section{Acknowledgments}

The authors acknowledge support from NCI P01 55164, from the George and Barbara Bush Leukemia Research Fund, the Anderson Chair for Cancer Treatment and Research at the University of Texas M.D. Anderson Cancer Center, the Ensign Professorship of Medicine at the Yale University School of Medicine, and the Hull Development Fund of the Yale Cancer Center.

\section{References}

1. Marlton P, Keating $M$, Kantarjian $H$, Pierce $S$, O'Brien S, Freireich EJ, Estey E. (1995) Cytogenetic and clinical correlates in AML patients with abnormalities of chromosome 16. Leukemia 9: 965-971.

2. Walker H, Smith FJ, Betts DR. (1994) Cytogenetics in acute myeloid leukemia. Blood Rev. 8: 30-36.

3. Maseki N, Miyoshi H, Shimizu K, Homma C, Ohki M, Sakurai M, Kaneko Y. (1993) The 8;21 chromosome translocation in acute myeloid leukemia is always detectable by molecular analysis using AML1. Blood 81: 1573-1579.

4. Symes PH, Williams ME, Flessa HC, Srivastava AK, Swerdlow SH. (1993) Acute promyelocytic leukemia with the pseudo-Chediak-Higashi anomaly and molecular documentation of $\mathrm{t}(15$; 17) chromosomal translocation. Am. J. Clin. Pathol. 99: 622-627.

5. Hoffmann L, Moller P, Pedersen BJ, Waage A, Pedersen M, Hirsch FR. (1995) Therapy-related acute promyelocytic leukemia with $\mathrm{t}(15 ; 17)$ (q22; q12) following chemotherapy with drugs targeting DNA topoisomerase II. A report of two cases and a review of the literature. Ann. Oncol. 6: 781788.

6. Baranger L, Baruchel A, Leverger G, Schaison G, Berger R. (1990) Monosomy-7 in childhood hemopoietic disorders. Leukemia 4: 345-349.

7. Stephenson J, Lizhen H, Mufti GJ. (1995) Possible co-existence of RAS activation and monosomy 7 in the leukemic transformation of myelodysplastic syndromes. Leuk. Res. 19: 741-748.

8. Vogler WR. (1992) Strategies in the treatment of acute myelogenous leukemia. Leuk. Res. 16: $1143-$ 1148.

9. Downing JR, Head DR, Curcio BA, et al. (1993) An AMLl/ETO fusion transcript is consistently detected by RNA-based polymerase chain reaction in acute myelogenous leukemia containing the $(8 ; 21)(\mathrm{q} 22 ; \mathrm{q} 22)$ translocation. Blood 81: 2860-2865.

10. Lo CF, Diverio D, D'Adamo F, Avvisati G, Alimena G, Nanni M, Alcalay M, Pandolfi PP, Pelicci PG. (1992) PML/RAR-alpha rearrangement in acute promyelocytic leukemias apparently lacking the $\mathrm{t}(15 ; 17)$ translocation. Eur. J. Haematol. 48: 173177.

11. Nucifora G, Dickstein JI, Torbenson V, Roulston D, Rowley JD, Vardiman JW. (1994) Correlation between cell morphology and expression of the AML1/ETO chimeric transcript in patients with acute myeloid leukemia without the $t(8 ; 21)$. Leukemia 8: 1533-1538.

12. de Greef G, Hagemeijer A, Morgan R, Wijsman J, Hoefsloot LH, Sandberg AA, Sacchi N. (1995) Identical fusion transcript associated with different breakpoints in the AMLl gene in simple and variant $\mathrm{t}(8 ; 21)$ acute myeloid leukemia. Leukemia 9: $282-288$. 
13. Reed JC. (1995) Bcl-2 family proteins: regulators of chemoresistance in cancer. Toxicol. Lett. 155: 82-84.

14. Korsmeyer SJ. (1995) Regulators of cell death. Trends Genet. 11: 101-105.

15. Craig RW. (1995) The bcl-2 gene family. Semin. Cancer Biol. 6: 35-43.

16. Yin XM, Oltval ZN, Korsmeyer SJ. (1994) BHl and $\mathrm{BH} 2$ domains of $\mathrm{Bcl}-2$ are required for inhibition of apoptosis and heterodimerization with Bax [see comments]. Nature 369: 321-323.

17. Boise LH, Gonzalez GM, Postema CE, et al. (1993) $\mathrm{Bcl}-\mathrm{X}$, a bcl-2-related gene that functions as a dominant regulator of apoptotic cell death. Cell 74: 597-608.

18. Kozopas KM, Yang T, Buchan HL, Zhou P, Craig RW. (1993) MCLl, a gene expressed in programmed myeloid cell differentiation, has sequence similarity to BCL2. Proc. Natl. Acad. Sci. USA 90: 3516-3520.

19. Miyashita T, Reed JC. (1995) Tumor suppressor p53 is a direct transcriptional activator of the human bax gene. Cell 80: 293-299.

20. Park JR, Bernstein ID, Hockenbery DM. (1995) Primitive human hematopoietic precursors express Bcl-x but not Bcl-2. Blood 86: 868-876.

21. Chittenden T, Harrington EA, O'Connor R, Flemington C, Lutz RJ, Evan GI, Guild BC. (1995) Induction of apoptosis by the Bcl-2 homologue Bak. Nature 374: 733-736.

22. Farrow SN, White JH, Martinou I, et al. (1995) Cloning of a bcl-2 homologue by interaction with adenovirus E1B 19K. Nature 374:731-733.
23. Karsan A, Yee E, Kaushansky K, Harlan JM. (1996) Cloning of human bcl-2 homologue: Inflammatory cytokines induce human Al in cultured endothelial cells. Blood 87: 3089-3096.

24. Kiefer MC, Brauer MJ, Powers VC, Wu JJ, Umansky SR, Tomei LD, Barr PJ. (1995) Modulation of apoptosis by the widely distributed Bcl-2 homologue Bak. Nature 374: 736-739.

25. Lin EY, Orlofsky A, Berger MS, Prystowsky MB. (1993) Characterization of Al, a novel hemopoietic-specific early-response gene with sequence similarity to bcl-2. J. Immunol. 151: 1979-1988.

26. Yang E, Zha J, Jockel J, Boise LH, Thompson CB, Korsmeyer SJ. (1995) Bad, a heterodimeric partner for Bcl-XL and Bcl-2, displaces Bax and promotes cell death. Cell 80: 285-291.

27. Maung ZT, MacLean FR, Reid MM, Pearson AD, Proctor SJ, Hamilton PJ, Hall AG. (1994) The relationship between bcl-2 expression and response to chemotherapy in acute leukaemia. $B r . J$. Haematol. 88: 105-109.

28. Keith FJ, Bradbury DA, Zhu YM, Russell NH. (1995) Inhibition of bcl-2 with antisense oligonucleotides induces apoptosis and increases the sensitivity of AML blasts to ara-C. Leukemia 9: 131138.

29. Minn AJ, Rudin CM, Boise LH, Thompson C. (1995) Expression of bcl-xl can confer a multidrug resistance phenotype. Blood 86: 1903-1910.

30. Dole MG, Jasty $R$, Cooper MJ, Thompson CB, Nunez G, Castle VP. (1995) Bcl-xl is expressed in neuroblastoma cells and modulates chemotherapy-induced apoptosis, Cancer Res. 55: 2576-2582. 